\title{
Biosensors for Cellular Imaging on the Base of Colloidal CdMns Nanoparticles
}

\author{
A. I. Savchuk ${ }^{1, *}$, M. M. Marchenko ${ }^{1}$, T. A. Savchuk ${ }^{1}$, S. A. Ivanchak ${ }^{1}$, \\ V. I. Fediv², I. S. Davydenko², and D. I. Ostafiychuk ${ }^{2}$ \\ ${ }^{1}$ Department of Physics of Semiconductors and Nanostructures, Chernivtsi National University, Chernivtsi 58012, Ukraine \\ ${ }^{2}$ Department of Biophysics and Medical Informatics, Department of Pathomorphology and Forensic Medicine, \\ Bukovina State Medical University, Chernivtsi 58002, Ukraine
}

(Received: 4 June 2009. Accepted: 21 December 2009)

\begin{abstract}
Semiconductor nanoparticles have rapidly found a broad range of applications as optical imaging agents. The presented paper describes the growth, analysis of optical spectra and study of cellular imaging of Mn-doped cadmium sulfide nanoparticles. Semimagnetic semiconductor nanoparticles CdMnS are characterized by high brightness, improved photostability and multicolor size- and structure-dependent light emission in the visible range of spectra. They are emerging as a new class of fluorescent reporters with properties and applications that are not available with traditional organic dyes. In order to obtain nanosensors different surfactants, growth factors and conditions such as surfactant concentrations, ionic strength, precursor concentration have been varied. Formation of nanoparticles was monitored by optical methods. The average nanoparticle size was estimated by optical spectra. For cellular research histological section of a placental tissues and liver tissues has been used. This procedure is possible only after cell fixation and permeabilization treatment is needed to allow the nanosensors to enter inside the cell.
\end{abstract}

Keywords: Biosensor, Semimagnetic Semiconductor, CdMnS, Nanoparticle, Optical Density, Cell.

\section{INTRODUCTION}

Nanotechnology and nanoscale materials are a new and exciting field of research. The inherently small size and unusual optical, magnetic, catalytic, and mechanical properties of nanoparticles not found in bulk materials permit the development of novel devices and applications previously unavailable. One of the earliest applications of nanotechnology that has been realized is the development of improved chemical and biological sensors. ${ }^{1,2}$

Fluorescence sensing is among the most widely used approaches suggested for reporting about sensor-target interaction. This method possesses such distinguishing features: ultra-high sensitivity, high speed of response, high spatial resolution, non-destructive and non-invasive. In a broader sense, fluorescence allows the visualization of sensor-target interactions with a high spatial resolution. Cell imaging is important field in the application of fluorescence emitters in the form of nanosensors. Organic

\footnotetext{
${ }^{*}$ Corresponding author; E-mail: savchuk@chnu.cv.ua
}

fluorescent compounds such as fluorescent proteins are extensively used to study molecular and cellular processes. Despite their considerable advantages in live cell imaging, organic fluorophores are subject to certain limitations (selfquenching at high concentrations, susceptibility to photobleaching, short-term aqueous stability, narrow absorption windows coupled to broad red-tailed emission spectra via small Stokes shifts often result in spectra overlap, and short excited state fluorescent lifetimes). ${ }^{3-6}$

Semiconductor nanocrystals (quantum dots, QDs) have been proposed as alternative fluorescent labels that overcome many of these limitations, open up new possibilities especially for biomolecular and cellular imaging. Since their introduction as labels in cellular imaging in $1998,^{7,8}$ multiple reports have shown the strengths of these nanoprobes for tagging and imaging applications in biological systems. This strength is based on the impressive photostability of quantum dots which guarantees the observation of biomolecules over longer time periods, on their broad excitation and narrow size-dependent emission properties, large extinction coefficients, resistance 
to photobleaching, long fluorescence lifetime, and sizetunable emission. ${ }^{9}$ Depending on their material and their shape, nanocrystals can possess many varied properties.

As QDs' incompatibility with biomolecules, due to highly hydrophobic and toxic properties, the first step towards biomolecular and cellular imaging is to functionalize their hydrophobic surfaces by amphiphilic ligands. These coated-QDs systems (nanosensors) are considered as nano-scale hybrid systems that integrate recognition and reporting functionalities and their support matrix for the detection and quantitative analysis of target binding. ${ }^{10-18}$

The presence of localized magnetic ions in a semiconductor alloy leads to exchange interactions between $\mathrm{sp}$ band electrons and the $\mathrm{Mn}^{2+} \mathrm{d}$ electrons. It plays a double role in determining optical properties: (1) the bandgap of the compound is altered depending upon the concentration of manganese ions; (2) the $3 \mathrm{~d}$ levels of transition metal ions are located in the bandgap region and $\mathrm{d}-\mathrm{d}$ transitions dominate the spectrum. New possibilities in the field of colour biosensing are expected due to emitting transitions connected with $\mathrm{Mn}^{2+}$ ions.

Cells or tissue for labeling, prepared appropriately depending on the application (QDs can be used to tag live cells, label cell-surface proteins, or label fixed cells or tissue sections).

We here propose manganese ion-doped cadmium sulfide nanoparticles as a promising new class of biological fluorescent labels for fixed cells or tissue sections of placenta and liver. The 2-mercaptoethanol-CdMnS nanoparticles and cetyl trimethyl ammonium bromide (CTAB)-CdMnS nanoparticles nanosensors were used for labeling specific cellular proteins.

\section{EXPERIMENTAL DETAILS}

\subsection{Synthesis}

Aqueous syntheses of nanoparticles offer many benefits for biological studies. The synthesis of particles in a solution occurs by chemical reactions resulting in the formation of nuclei and subsequent particle growth. In the precipitation of multicomponent material, special attention is needed to control co-precipitation conditions in order to achieve chemical homogeneity of the final product. This is due to the fact that different ions often precipitate under different conditions of $\mathrm{pH}$ and temperature having different solubility product constants. The growth of nanocrystals depends on a number of parameters, such as the surface energy of the nanocrystal, the concentration of free species in a solution, and the nanocrystal size, and thus the nanocrystal growth can be controlled by considering these parameters. A theoretical consideration of the probability of chemical reactions in the multicomponent system $\mathrm{Cd}^{2+}-\mathrm{Mn}^{2+}-\mathrm{S}^{2-}-\mathrm{H}_{2} \mathrm{O}$ in case of the synthesis of nanoparticles has shown that the optimal ranges of a change of the molar concentration of precursors and the $\mathrm{pH}$ value of the medium are within the limits of $\left(10^{-4}-10^{-2}\right) \mathrm{mol} / \mathrm{L}$ and (3-5) respectively.

Van der Waals interactions favor interparticle aggregation, however, the presence of the stable organic coating prevents the inorganic cores of neighboring nanocrystals from touching each other, thus resulting in a solution of well-dispersed particles. Surfactants are crucial for a controlled growth and stability of nanocrystals. They bind to the surface of the growing crystals and, in addition, they form complexes with the atomic species in a solution, thus controlling the reactivity and the diffusion of the elemental species to the surface of the growing nanocrystals. Without them, the initial species would bind rapidly to each other, resulting in an uncontrolled growth of nanocrystals.

The synthesis of $\mathrm{CdMnS}$ nanoparticles was realized in the multicomponent system $\mathrm{CdCl}_{2}-\mathrm{MnCl}_{2}-\mathrm{Na}_{2} \mathrm{~S}-\mathrm{H}_{2} \mathrm{O}$ surfactant at room temperature. The concentrations of salt solutions of $\mathrm{CdCl}_{2}, \mathrm{MnCl}_{2}, \mathrm{Na}_{2} \mathrm{~S}$ and the $\mathrm{pH}$ value were selected with regard to theoretical considerations. Particles with various properties were obtained by varying either the $\mathrm{pH}$ value, or the surfactant concentration and the $\left[\mathrm{CdCl}_{2}\right]$ : $\left[\mathrm{Na}_{2} \mathrm{~S}\right]$ molar ratio, $\left[\mathrm{CdCl}_{2}\right]:\left[\mathrm{MnCl}_{2}\right]$ molar ratio. In order to keep the system in the focusing regime (the formation of nearly monodisperse nanocrystals) for a long time, it is possible to perform additional slow injections of precursors during the growth, effectively keeping the concentration of free species in a solution above a critical threshold.

The stability of the nanoparticles is ensured using a surface complexation with 0.1 molar solution of CTAB and 0.05 molar solution of 2-mercaptoethanol.

\subsection{Characterization Methods}

Optical monitoring of the formation of nanoparticles was controlled by a step-by-step measurement of the absorption spectrum, depending on the concentration of precursors in the range of $\left(10^{-4}-10^{-2}\right) \mathrm{mol} / \mathrm{L}$. Optical absorption measurements were carried out on a set-up whose key elements were a grating monochromator for wavelengths (200-2200) $\mathrm{nm}$ in the presence of a UV source of light.

\subsection{Fixed Tissue and Method of Testing}

Histological specimens of the human placenta with the term of pregnancy 32-40 weeks and histological liver specimens of dead fetuses and dead newborns were investigated.

The authors used a technique of tissue treatment which minimally changes its chemical and structural properties, in particular, it permits to preserve suitable to be detected the majority of antigens ${ }^{19}$ and sulfydryl groups of protein molecules with a possibility of their prolonged conservation in paraffin blocks. The basic principles of the 
mentioned technique of tissue treatment consist in the following:

(1) tissue fixation in a buffered $10 \%$ formalin solution by Lilli's phosphate buffer $(\mathrm{pH}=7.0)$ during 22 hours (a prolonged time of formalin treatment results in artefacts of the structure of the majority of antigens),

(2) accelerated dehydration in the initial battery of spirits,

(3) paraffin infiltration at a temperature of $58{ }^{\circ} \mathrm{C}$ (higher temperature readings may cause undesirable changes of the structure of antigens).

Histological sections $5 \mu \mathrm{m}$ thick were obtained by means of a sliding microtome. All the adduced results of the study belong exclusively to the above mentioned tissue treatment.

De-embedding of histological sections was performed by means of a standard technique, using xylol, ethanol and water. Further treatment by nanosensors and a shortterm wash-out permitted a possibility of photoluminescence imaging of the structures of the tissues under study. The trial was performed with the aid of the fluorescence microscope and a digital camera.

\section{RESULTS AND DISCUSSION}

Figure 1 shows the optical density spectra of CdMnS nanoparticles for varying salt concentrations (monitoring of growth) and different surfactants. The average size of particles was calculated ${ }^{20,21}$ to be $2.5 \mathrm{~nm}$ in diameter for mercapto-coated QD and $12 \mathrm{~nm}$ in diameter for CTAB-coated QD. The surface charge of CdS nanoparticles can be changed by adjusting the $\left[\mathrm{CdCl}_{2}\right] /\left[\mathrm{Na}_{2} \mathrm{~S}\right]$ ratio. When the amount of $\mathrm{Na}_{2} \mathrm{~S}$ molecules was more superfluous than that of $\mathrm{CdCl}_{2}$ molecules, the surface of $\mathrm{CdMnS}$ nanoparticles was surrounded by $\mathrm{S}^{2-}$ anions, and thus, the nanoparticles were negative.

The position of absorption peaks remained almost unchanged at $3.4 \mathrm{eV}$ and at $2.95 \mathrm{eV}$ regardless of the precursors concentrations in the range $5 \cdot 10^{-4}-5 \cdot 10^{-2} \mathrm{~mol} / \mathrm{L}$, therefore the size of the CdMnS nanoparticles was also invariable. But absorption peaks slightly increased with an increase of the amount of the precursor that is indicative of a growth of the concentration of nanoparticles.

Figure 2 shows the photoluminescence (PL) spectra of CTAB-coated CdMnS nanoparticles. It revealed two wide PL band. The peak at $\sim 440 \mathrm{~nm}$ is the near-band-edge luminescence, while the wider long-wavelength band is an overlap of the emission bands related to the transitions involving d-electrons of $\mathrm{Mn}^{2+}$ ions and the recombination via surface localized states. ${ }^{22}$

For biological imaging applications, biocompatibility is the most important challenge to make nanoparticles work as novel fluorescent probes.

Though nanoparticles are very small, their surface area is large enough for linking to multiple biomolecules. ${ }^{23}$ There are several ways to bind biomolecules to quantum dots.
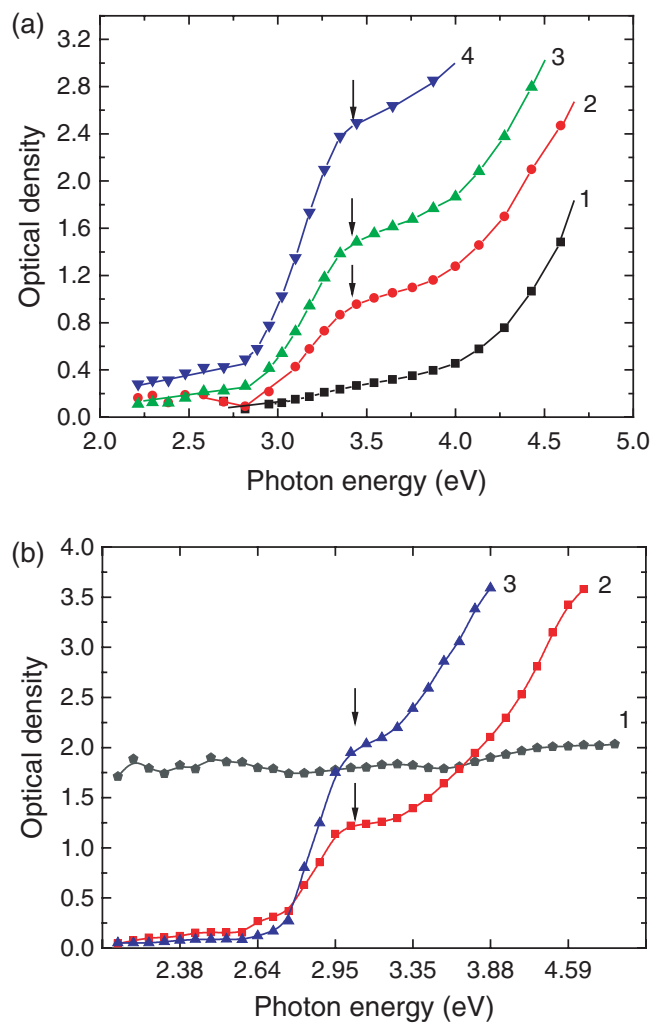

Fig. 1. Optical density as a function of photon energy for colloidal solution of (a) mercapto-coated CdMnS nanoparticles at $\mathrm{MnCl}_{2}$ concentration $1 \cdot 10^{-3} \mathrm{~mol} / \mathrm{L}$ (1-matrix (aqueous solution of mercaptoethanol $+\mathrm{CdCl}_{2}+$ $\left.\mathrm{MnCl}_{2}\right) ; 2,3$, 4-cycles of adding reagents $\left(\mathrm{Na}_{2} \mathrm{~S}\right.$ and $\left.\mathrm{CdCl}_{2}\right)$ with concentration respectively $10^{-3}, 2,5 \cdot 10^{-3}, 5 \cdot 10^{-3} \mathrm{~mol} / \mathrm{L}$ ) and (b) CTAB-coated $\mathrm{CdMnS}$ nanoparticles at $\mathrm{MnCl}_{2}$ concentration $5 \cdot 10^{-2} \mathrm{~mol} / \mathrm{L}(1-$ matrix (aqueous solution of $\mathrm{CTAB}+\mathrm{CdCl}_{2}+\mathrm{MnCl}_{2}$ ), 2, 3-cycles of adding reagents $\left(\mathrm{Na}_{2} \mathrm{~S}\right.$ and $\left.\mathrm{CdCl}_{2}\right)$ with concentration respectively $1,5 \cdot 10^{-3}, 2$, $\left.5 \cdot 10^{-3} \mathrm{~mol} / \mathrm{L}\right)$.

These involve either direct binding to the quantum dot surface or attachment via a stabilizing layer acting as a crosslinker between the ligand and reactive surface of the nanoparticle.

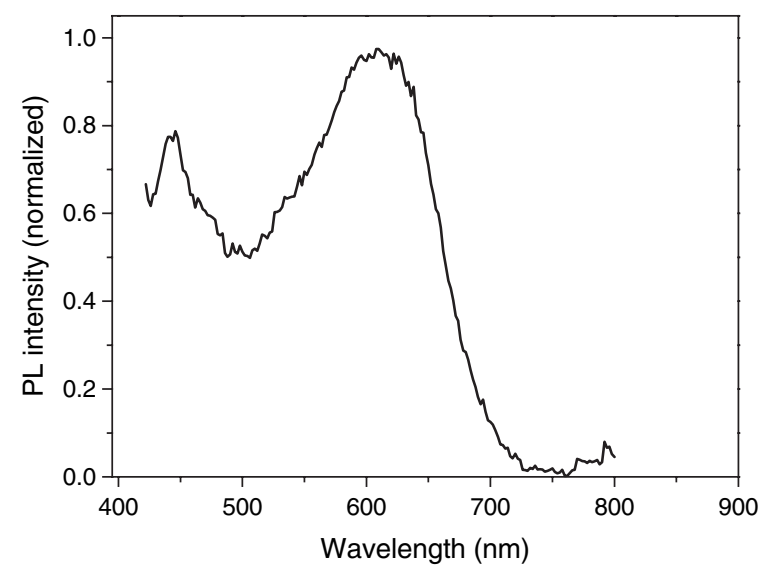

Fig. 2. Photoluminescence spectra of CTAB-coated CdMnS nanoparticles. 


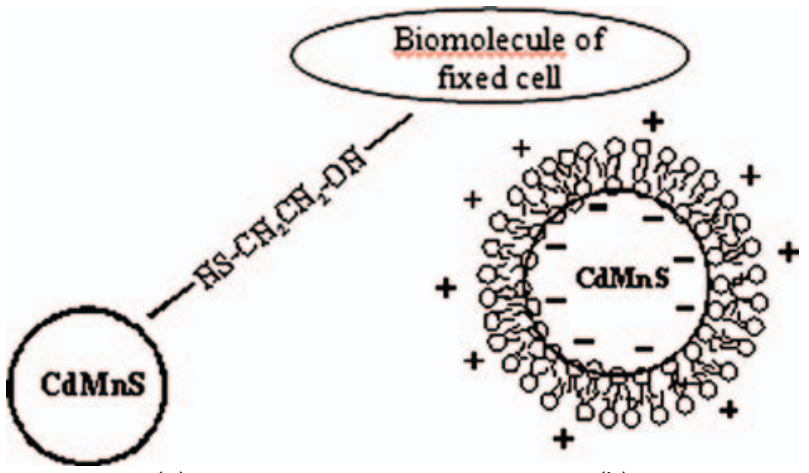

(a)

(b)

Fig. 3. Schematic representation of the (a) mercapto-coated CdMnS nanoparticles and (b) CTAB-coated CdMnS nanoparticles.

Nanosensors have two important properties: they are capable of molecular recognition and they can self-assemble. The self-assembly process dictates that nanosensors find their appropriate positions without any external driving force. Molecular recognition is a "key/lock" principle realized on a molecular scale. Receptor molecules (the lock) recognize certain nanosensors (the "key") with very high selectivity. Molecules of fixed tissue are used as a "lock". Two type of nanosensors, such as mercapto-coated QD and CTAB-coated QD are used as a "key".5

Bifunctional molecules 2-mercaptoethanol [HS- $\left(\mathrm{CH}_{2}\right)_{n}$ $\mathrm{OH}$ ] was used for the formation of mercapto-coated QD. Thiol (-SH)-containing molecules are often used to anchor functional groups on QDs surfaces (Fig. 3(a)). The binding of 2-mercaptoethanol occurs through the sulfur group at the molecule terminus, which adheres to $\mathrm{Cd}$ atoms on the CdMnS coating. The hydroxyl end of 2-mercaptoethanol provides water solubility and may also be reactive to biomolecules due to the intermolecular hydrogen bond.

In case of CTAB-coated QD bifunctional molecules a CTAB with both hydrophobic and hydrophilic ends were used. The head-group charge present on ionic surfactants results in a more complicated adsorption process when compared to nonionic amphiphiles. Ionic surfactant adsorption is particularly sensitive to the interactions of counter- and co-ions with the charged groups of the surface. Their polar head groups are functional moieties capable of donating electron pairs. Controlling the growth of nanocrystals is mainly dictated by their ability to form complexes with the free $\mathrm{Cd}$ species in a solution and to their binding ability to the $\mathrm{Cd}$ atoms on the surface of the nanocrystals. Adjustment of the solution $\mathrm{pH}$ may also affect the level of dissociation of surface groups and the overall ionic strength in the surfactant substrate system. All these factors have implications, not only for the surface excess, but also for the morphology of the surface aggregates formed. ${ }^{24}$

An interaction of the hydrophilic part of CTAB with negatively-charged ions of the nanoparticle surface seems most probable with further stabilization of this system at the expense of the formation of a double layer (Fig. 3(b)). Biomolecules, in this case, interact with a formed nanosensor owing to the electrostatic interaction as well as to Van
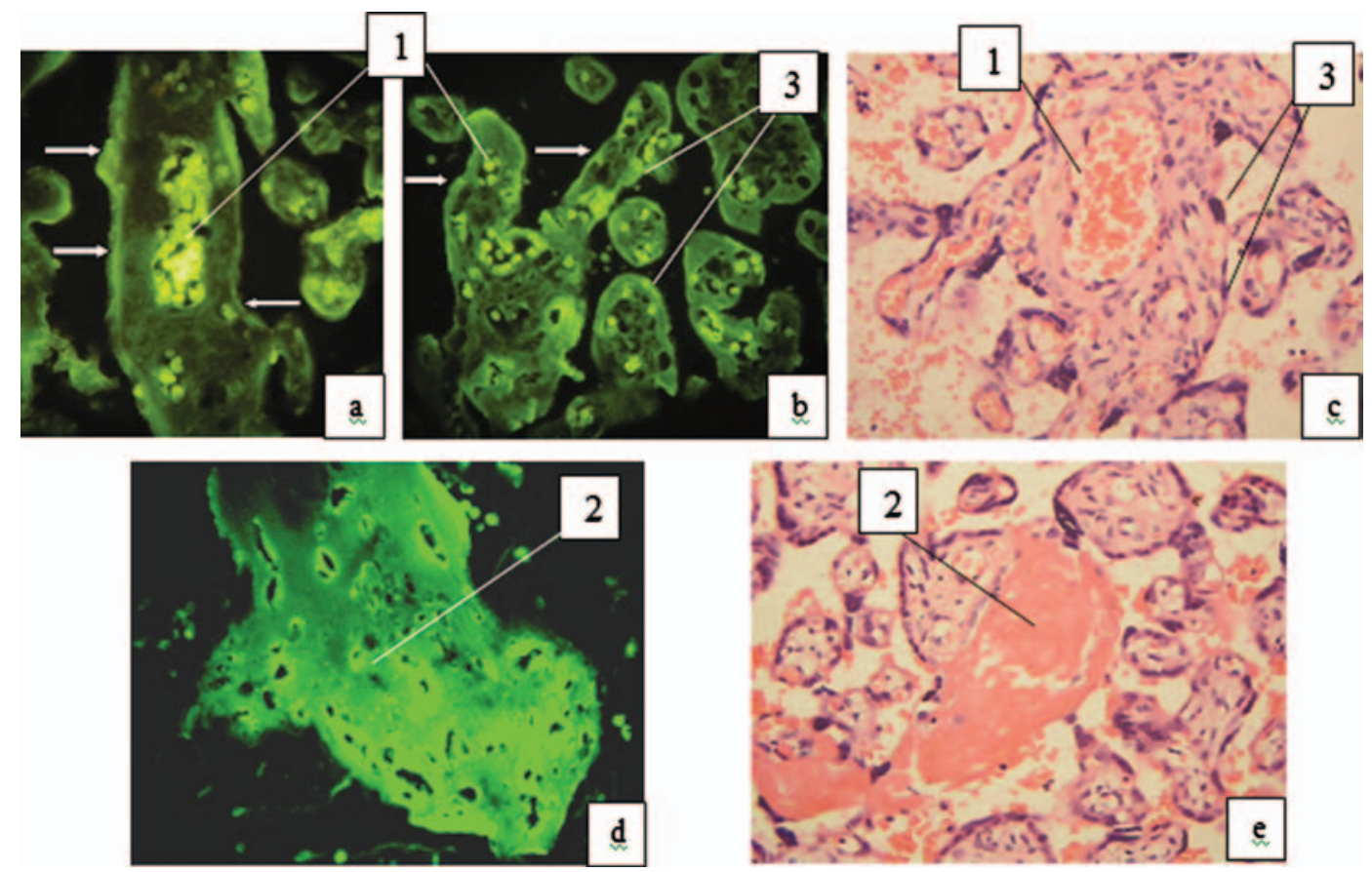

Fig. 4. Photoluminescent image of some structures of the placental chorial tree obtained upon treating histologic specimens with (a)-(c) mercaptocoated nanoparticles and (d), (e) with hematoxylin and eosin of the placenta of a person with a 32-40 week term of pregnancy. (1-erythrocyte, 2-intervillous fibrinoid, 3-syncytiotrophoblast). 
der Waals interaction of the hydrophobic part of CTAB due to a dynamic exchange.

Figure 4 represents a photoluminescent image of some structures of the placental chorial tree obtained upon treating histologic specimens with nanoparticles of the placenta of a person with a 32-40 week term of pregnancy.

The most intensive luminescence was observed in the maternal erythrocytes and fetal erythrocytes (Figs. 4(a, b)), as well as in the intervillous fibrinoid (Fig. 3(c)). Less intensive, but still well defined luminescence is marked in the syncytiotrophoblast and endotheliocytes of the blood vessels of the chorial villi which are preliminarily identified as stromal cells, taking into account the dimensions, forms and the specific characteristics of the location (most reliably, with due regard for the term of pregnancyfibroblasts). The spectral range of imaging was found in the region of the green color (about $500 \mathrm{~nm}$ ).

The experiments, involving digital photographing of luminescent images with different exposures (under other equal conditions of photographing) permitted to reveal the fact that the luminescence of erythrocytes was due to the luminescence of their membrane (Figs. 4(a, b)) and the luminescence of the erythrocytic cytoplasm is perceived as a consequence of light scattering.

Luminescence of various intensity was observed in the intervillous fibrinoid and it was characterized by a certain consistent pattern that was particularly traced in a canalized fibrinoid. The sites on the surface of the fibrinoid and the thin wall of its polymorphous canals where as a rule, according to immunohistochemical findings, adhesive molecules are concentrated, in particular integrin- $\alpha-\mathrm{X}-\beta 2$ (CD11c/CD18) (Fig. 3(c)) scintillated most intensively. ${ }^{25}$ This luminescence differed by the spectrum from other sites of the fibrinoid and were in the vicinity of $560 \mathrm{~nm}$. In order to eliminate subjectivity, while evaluating the color of staining a spectral computer analysis of the image sites in the RGB system was implemented. A similar luminescence in the form of a thin strip was also marked on the surface of syncytiotrophoblast (in Figs. 4(a, b) —indicated by white arrows) that also corresponds to the immunohistochemical location of the said integrin- $\alpha-\mathrm{X}-\beta 2{ }^{25}$

The luminescence of the syncytiotrophoblast was irregular both along the perimeter of each chorial villus and in the cytoplasm of the syncytiotrophoblast from its apical to basal portions that is, evidently, accounted for by a change of the concentration of different substances and is a normal value for this particular structure. ${ }^{26}$ At the same time, the chorial villi are characterized by a regularity which consists in the fact that the luminescence of the syncytiotrophoblast has always been more intensive in the basal rather than apical portions (with the exception of the very surface of the trophoblast, the latter having been mentioned above).

The luminescence of the endothelium of the blood vessels of the chorial villi in comparison with the syncytiotrophoblast differed by higher stability on a vessel cross-section (Figs. 4(a, b)). The structure of the epithelium was seen particularly well in the vessels which didn't contain erythrocytes (Figs. 4(a, b)). No ruptures were marked between erythrocytes. It was explained by the fact that placentas with the physiological course of pregnancy were studied. In case of a pathological course of pregnancy disruptions between endotheliocytes occur and one can manage to diagnose them by this particular luminescence technique, that is the technique may be used as a diagnostic procedure as to a damaged endothelial tegument of the blood vessels.

It should be noted that the luminescence of a number of structures which are obligatory elements of the chorial

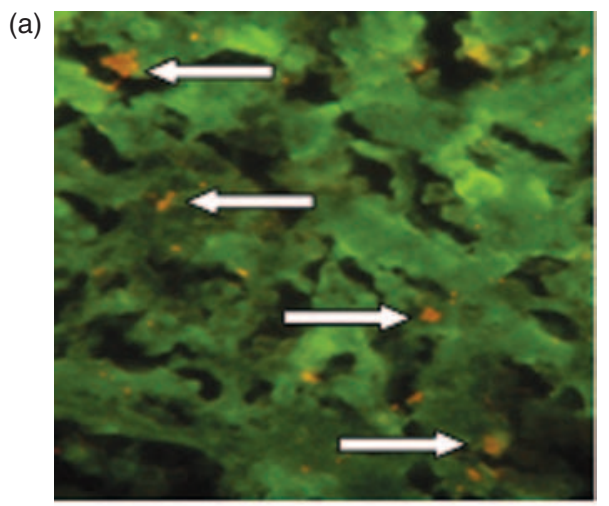

(b)

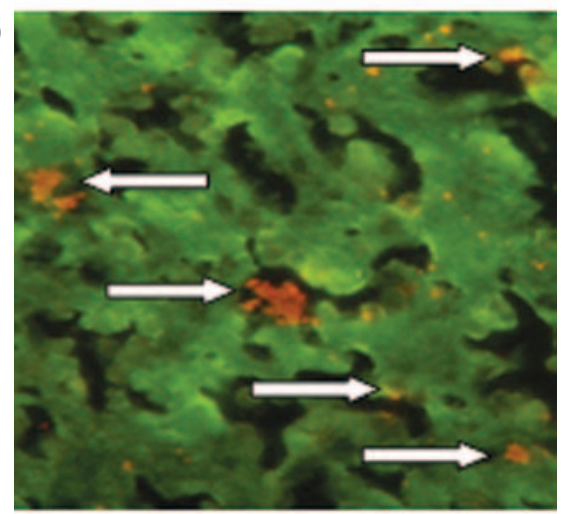

(c)

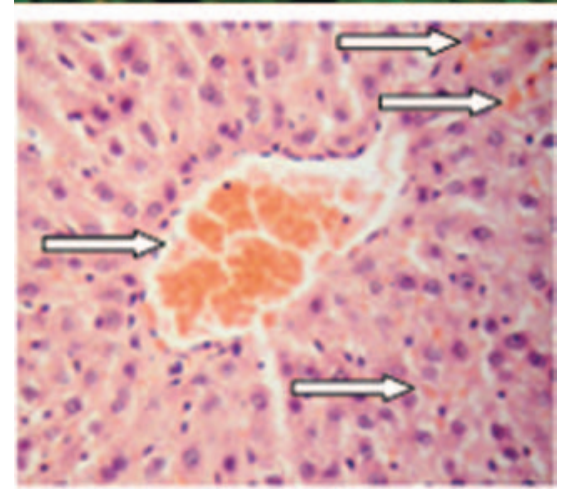

Fig. 5. Photoluminescent image of the structures of the liver upon treating histologic specimens with CTAB-coated nanoparticles of (a) dead fetuses, (b) dead neonales and (c) with hematoxylin of dead neonales. A bright red luminescence of objects (indicated by white arrows) denoted localizations of Kupffer's cells. 
villi was not marked. This, particularly, concerns the basic substance and the fibers of the connective tissue, subepithelial and subendothelial basal membranes which were not visualized in the studied histologic sections of the placental chorial villi.

The identification of microscopic structures of the placenta was realized with special regard for modern conceptions about the structure of the chorial villi and extravillous structures of this organ with the term of pregnancy-40 weeks. ${ }^{25}$ For the sake of comparison Figures $4(d, e)$ represents a histologic section of the placenta stained with hematoxylin and eosin that is most often used in histologic practice.

A study of histologic specimens of the liver of dead fetuses and dead newborns was also carried out (Fig. 5(a)). Green luminescence completely corresponded to the localization and form of the cells which were identified as hepatocytes and erythrocytes on the basis of their morphological signs. The spectral range of the image was in the region of the green color (nearly $500 \mathrm{~nm}$ ).

A bright red luminescence of objects (indicated by white arrows) was also observed. Taking into account their localizations (the wall of the sinusoids), dimensions, their number in a unit of the square area of the section, contour polymorphism, these particular objects correspond to Kupffer's cells (stellate reticuloendotheliocytes) and their cellular processes.

To eliminate subjectivity, when evaluating the color of staining, a spectral computerized analysis of images in the RGB system was implemented.

The identification of Kupffer's cells was realized with regard for modern conceptions of the liver structure ${ }^{27}$ and comparing with the images of the histological sections of the liver stained with hematoxylin and eosin (Fig. 5(b)). This particular technique makes it possible to implement a morphologic evaluation of the liver condition based on the number and localization of Kupffer's cells.

\section{CONCLUSIONS}

The CdMnS nanoparticles have been prepared in the multicomponent system $\mathrm{CdCl}_{2}-\mathrm{MnCl}_{2}-\mathrm{Na}_{2} \mathrm{~S}-\mathrm{H}_{2} \mathrm{O}$-surfactant at room temperature. It has been revealed that an increase of the concentration of salts in a solution in the range of $5 \cdot 10^{-4}-5 \cdot 10^{-2} \mathrm{~mol} / \mathrm{L}$ leads to an elevation of the concentration of nanoparticles in the colloidal solution and doesn't influence on the size of nanoparticles. The suggested luminescence technique, employing nanosensors permits to determine differentially placental tissue structures in histological sections and reveal Kupffer's cells in histological sections of the liver which may be used for diagnostic procedures. An outlook of further studies consists in a detailed research of a relationship between luminescence characteristics and pathologic changes of a tissue, the nature of chemical bonds among the structures of biological tissues with nanosensors have been ascertained.

Acknowledgments: This work has been supported in part by grant from Ministry of Education and Science of Ukraine.

\section{References and Notes}

1. J. Ji, J. A. Schanzle, and M. B. Tabacco, Anal. Chem. 76, 1411 (2004).

2. F. S. Ligler, C. R. Taitt, L. C. Shriver-Lake, K. E. Sapsford, Y. Shubin, and J. P. Golden, Anal. Bioanal. Chem. 377, 469 (2003).

3. J. Zhang, R. E. Campbell, A. Y. Ting, and R. Y. Tsien, Nat. Rev. Mol. Cell Biol. 3, 906 (2002).

4. R. P. Haugland, Handbook of Fluorescent Probes and Research Products; Molecular Probes, Eugene, OR (2002).

5. T. Pellegrino, S. Kudera, T. Liedl, A. M. Javier, L. Manna, and W. J. Parak, J. Small 1, 49 (2005).

6. X. Gao, Y. Y. Cui, R. M. Levenson, L. W. K. Chung, and S. Nie, Nat. Biotechnol. 22, 969 (2004).

7. M. J. Brunchez, M. Moronne, P. Gin, S. Weiss, and A. P. Alivisatos, Science 281, 2013 (1998).

8. W. C. W. Chan and S. Nie, Science 281, 2016 (1998).

9. A. Fu, W. Gu, C. Larabell, and A. P. Alivisatos, Current Opinion in Neurobiology 15, 568 (2005).

10. L. Zhu, S. Ang, and W.-T. Liu, Applied and Environmental Microbiology 70, 597 (2004).

11. N. Kaji, M. Tokeshi, and Y. Baba, Anal. Sci. 23, 21 (2007).

12. A. P. Alivisatos, Nat. Biotechnol. 22, 47 (2004).

13. I. L. Medintz, H. T. Uyeda, E. R. Goldman, and H. Mattoussi, Nat. Mater. 4, 435 (2005).

14. X. Michalet, F. F. Pinaud, L. A. Bentolila, J. M. Tsay, S. Doose, J. J. Li, G. Sundaresan, A. M. Wu, S. S. Gambhir, and S. Weiss, Science 307, 538 (2005)

15. B. Dubertret, P. Skourides, D. J. Norris, V. Noireaux, A. H. Brivanlou, and A. Libchaber, Science 298, 1759 (2002).

16. J. K. Jaiswal, H. Mattoussi, J. M. Mauro, and S. M. Simon, Nat. Biotechnol. 21, 47 (2003).

17. W. C. Chan, D. Maxwell, X. Gao, R. Bailey, M. Han, and S. Nie, Curr. Opin. Biotechnol. 13, 40 (2003).

18. M. Dahan, S. Levi, C. Luccardini, P. Rostaing, B. Riveau, and A. Triller, Science 302, 442 (2003)

19. R. Burns (ed.), Immunochemical protocols, Methods in Molecular Biology, 3rd edn., Humana Press Inc. (2005), Vol. 295, p. 317.

20. P. E. Lippens and M. Lannoo, Phys. Rev. B 39, N15 (1989).

21. Q. Pang, B. C. Guo, C. L. Yang, S. H. Yang, M. L. Gong, W. K. Ge, and J. N. Wang J. Cryst. Growth 269 (2004).

22. A. Nag, S. Sapra, S. S. Gupta, A. Prakash, A. Ghangrekar, N. Periasamy, and D. D. Sarma, Bull. Mater. Sci. 31 (2008).

23. M. J. Murcia and C. A. Naumann, Nanotechnologies for the Life Sciences 1, 1 (2005).

24. R. Atkin, V. S. J. Craig, E. J. Wanless, and S. Biggs, Adv. Colloid Interface Sci. 103, 219 (2003).

25. K. Ley (ed.), Adhesion Molecules: Function and Inhibition, Birkhäuser Verlag (2007).

26. K. Benirschke, P. Kaufmann, and R. N. Baergen, Pathology of the Human Placenta, 5th edn., Springer, New York (2006).

27. W. Kuehnel, Color Atlas of Cytology, Histology, and Microscopic Anatomy, 4th edn., Thieme, Stuttgart-New York (2002). 